O.E. Villamayor and D. Zelinsky

Nagoya Math. J.

Vol. 35. (1969), 83-98

\title{
GALOIS THEORY WITH INFINITELY MANY IDEMPOTENTS ${ }^{1)}$
}

\author{
O.E. VILLAMAYOR AND D. ZELINSKY
}

\section{Introduction.}

In 1942 Artin proved the linear independence, over a field $S$, of distinct automorphism of $S$; in other words if $G$ is a finite group of automorphisms of $S$ and $R$ is the fixed field, then $\operatorname{Hom}_{R}(S, S)$ is a free $S$-module with $G$ as basis. Since then, this last condition (" $S$ is $G$-Galois") or its equivalents have been used as a postulate in all the Galois theories of rings that are not fields, for example by Dieudonné, Jacobson, Azumaya and Nakayama for noncommutative rings and then in $[A G$, Appendix] and [CHR] for commutative rings. When $S$ has no idempotents but 0 and 1 , [CHR] proves that the ordinary fundamental theorem of Galois theory holds with no real change from the classical, field case.

If the rings have finitely many idempotents, the $G$-Galois condition prevents the "Galois group" $G$ from being the full automorphism group, but $[C H R]$ provides a Galois theory pairing all subgroups of $G$ with certain separable subalgebras. In a sense this is a study of the group $G$ as a transformation group on a commutative ring $S$. In [VZ] we presented a different Galois theory, oriented toward the rings rather than the groups, pairing all separable $R$-subalgebras of $S$ with some subgroups of the full automorphism group of $S$ over $R$. The present paper contains the same Galois theory, with no hypotheses at all on idempotents. The technique uses Pierce's representation [P] of the ground ring $R$ as the global cross sections of a sheaf of rings that have no nontrivial idempotents, so that at each point $x$ of the base space we have a ring extension of $R_{x}$ to which [VZ] applies.

In order to carry out this program, the $G$-Galois condition is too restrictive. Our hypothesis, besides the natural finite generation, projectivity,

Received July 13, 1968

1) This research was made possible by N.S.F. Grant GP-1649, Travel Grant GP-8231, and the Conference on Rings and Modules at Oberwolfach, March 1968. 
and separability of $S$ over $R$, can be phrased in three equivalent ways: $R$ is the fixed ring of some finite set (equivalently, group) of automorphisms of $S$; the $S$-module $\operatorname{Hom}_{R}(S, S)$ is generated (not necessarily freely) by automorphisms of $S ; R$ is a finite product $\Pi R_{i}$ of rings such that the corresponding direct factor of $S$ is $G$-Galois over $R_{i}$ for each $i$. The last hypothesis was called to our attention by H. F. Kreimer after this manuscript was prepared; he used it to prove theorems similar to ours [K]. Using our techniques, we have appended a proof that it is equivalent to the other two, at the end of section 3 .

\section{The Boolean spectrum.}

Pierce $[P]$ defined, for each commutative ring $R$, a sheaf of rings $\mathscr{R}$ over a totally disconnected compact Hausdorff space $X$ in such a way that $R$ is the ring of global cross sections of $\mathscr{R}$. We recast his definition slightly in the next few pages. Let $B(R)=$ the Boolean ring consisting of all idempotents of $R$.

(2. 1) Definition. The Boolean spectrum of $R$ is the Stone space $X=$ Spec $B(R)$ consisting of all prime (equivalently, for Boolean rings, maximal) ideals of $B(R)$.

It is possible to describe $X$ without reference to $B(R)$ :

(2. 2) A point $x$ in $X$ is a collection of idempotents in $R$ with the properties

(2. 2a) For every idempotent $e$ in $R$, either $e \in x$ or $1-e \in x$ but not both;

(2. 2b) If $e$ and $f$ are idempotents in $R$, then $e f \in x$ if and only if $e \in x$ or $f \in x$.

For each element $e$ in $x$, there is a neighborhood of $x$, namely $U_{e}=$ $\{y \in X \mid e \in y\}$. These neighborhoods form a base of the open sets. Notice $U_{e} \subset U_{f}$ if and only if $e \geq f$, that is $e f=f$.

From this description, we deduce a continuous map

(2. 3) $\phi:$ Spec $R \rightarrow X, \phi(p)=$ the set of idempotents in $p$.

Since $p$ is a prime ideal in $R$ and since $e(1-e)=0$, it is clear that $\phi(p)$ satisfies (2.2a) and (2.2b), and so is a point of $X$. Since $\phi^{-1}\left(U_{e}\right)=$ $\{p \in \operatorname{Spec} R \mid e \in p\}=\{p \in \operatorname{Spec} R \mid 1-e \notin p\}$ and this is a basic open set, $\phi$ is continuous. 
Note that $0 \in x$ and $1 \notin x$, for otherwise (2. 2a) implies $0 \notin x$ and $1 \in x$ and (2. 2b) then implies the contradiction $0=1 \cdot 0 \in x$. From this and (2. 8) below, we deduce that the $R$-ideal $R x$ generated by $x$ is proper for each $x$ (if $1 \in R x$ then $1=r e$ for some $e \in x$ and $1(1-e)=0,1=e \in x$ contradicting the definition of $x$ ), hence contained in some maximal ideal $p$ of $R$. Thus $\phi(p)=x$ and $\phi$ is surjective.

The following is another version of $X$ which, however, we shall not use explicitly.

(2. 4) $X$ is homeomorphic to the identification space one gets from Spec $R$ by identifying each connected component to a point. The homeomorphism is induced by $\phi$.

Proof. We first show that, for each $x \in X, \phi^{-1}(x)$ is a connected component of Spec $R$. If $V$ is any connected set in Spec $R, \phi(V)$ is connected in the totally disconnected space $X$, and hence is a single point. This means that $\phi^{-1}(x)$ is a union of connected components. We need only show $\phi^{-1}(x)$ is connected. The homomorphism $R \rightarrow R_{x}=R / R x$ induces a continuous map Spec $R_{x} \rightarrow$ Spec $R$ which merely associates to each prime ideal in $R_{x}$ its inverse image in $R$. From the definition of $\phi$, we see that the image of this map is $\phi^{-1}(x)$. Since Spec $R_{x}$ is connected (2.13), so is $\phi^{-1}(x)$.

This shows that $X$ is in one-to-one correspondence with the identification space. To show that the topology of these two spaces agree, we need to show that $\phi$ is continuous and closed. Its continuity has already been mentioned. Now assume $C$ is closed in Spec $R$. Then $C=\{p \mid p \supset I\}$ for some ideal $I$ in $R$. We see that $\phi(C)=\{x \in X \mid$ for some $p$ in Spec $R$, $p \supset R x+I\}=\{x \mid R x+I \neq R\}$. The complement of $\phi(C)$ is $\{x \mid R x+I=R\}$ and this is open, because $R x+I=R$ means $r e+i=1$ for some $r, e$ and $i$ in $R, x$ and $I$ respectively (see (2.8)); but then this same equation says $R y+I=R$ for every $y$ containing $e$, and this set of $y$ 's is a neighborhood of $x$.

Pierce's sheaf $\mathscr{R}$ over $X$ is then the direct image, under $\phi$, of the standard sheaf $\mathscr{O}(R)$ of local rings over Spec $R$. In other words, for every open set $U$ in $X$, the ring of cross-sections of $R$ over $U$ is defined to be $\Gamma\left(\phi^{-1}(U), \mathscr{O}(R)\right)$, where $\Gamma$ is the usual cross-section functor. In particular, we computed $\phi^{-1}\left(U_{e}\right)$ above; $\Gamma\left(U_{e}, \mathscr{R}\right)$ is the ring of fractions $S^{-1} R$ where $S$ is the multiplicatively closed set generated by $1-e$. Since $1-e$ is idempotent, we have 


$$
\Gamma\left(U_{e} \mathscr{R}\right)=\{(1-e)\}^{-1} R=R / R e .
$$

In particular, if $e=0$, we get $\Gamma(X, \mathscr{R})=R,[P, 4$. 4].

We can also describe the single stalks of $\mathscr{R}$ as both rings of fractions of $R$ and homomorphic images of $R$ : For each point $x$ in $X, R_{x}=\lim _{\rightarrow} \Gamma(U, \mathscr{R})$, the direct limit taken over the directed set of neighborhoods $U$ of $x$. Using the formula (2.5), we quickly get

$$
\begin{aligned}
R_{x} & =S^{-1} R \text { where } S=\{1-e \mid e \in x\} \\
& =R / R x
\end{aligned}
$$

where, of course, $R x$ is the ideal of $R$ generated by the elements of $x$. Note that $R_{x}$ is flat over $R$.

In fact, Pierce works even with noncommutative rings. The same remarks we have just made will also apply to this case, substituting the spectrum of the center of $R$ for $\operatorname{Spec} R$.

We need repeatedly the usual sheaf property: if two cross sections agree at a point, then they agree in a neighborhood of the point. However, for the sheaf $\mathscr{R}$ this is easily translated into simple algebraic terms and is quite special. We make this translation now and collect the major applications that we shall need.

Definition. Let $M$ be any $R$-module. Then

$$
\begin{aligned}
M_{x} & =M \otimes_{R} R_{x}=S^{-1} M \text { where } S=\{1-e \mid e \in x\} \\
& =M / M x
\end{aligned}
$$

If $a \in M$, then $a_{x}$ will denote the image of $a$ under $M \rightarrow M_{x}$. We remark that, if $M$ is finitely generated (resp. finitely presented, resp. faithful, resp. projective) as an $R$-module, then $M_{x}$ has the same property as an $R_{x}$-module. And if $M$ is a separable. $R$-algebra, then $M_{x}$ is a separable $R_{x}$-algebra.

If $g \in \operatorname{Hom}_{R}(M, M)$, we shall use $g_{x}$ to denote the $R_{x}$-module endomorphism of $M_{x}$ induced by $g$. This conflicts with the notation already introduced which would make $g_{x}$ an element of $\operatorname{Hom}_{R}(M, M)_{x}=\operatorname{Hom}_{R}$ $(M, M) \otimes_{R} R_{x}$. We shall adhere to the first version of $g_{x}$; in all our cases there is no real conflict, because $\operatorname{Hom}_{R}(M, M)_{x}$ will be identified with a subset of $\operatorname{Hom}_{R_{x}}(M, M)_{x}$. This is elucidated in the following proposition. 
(2. 7) The map $g \rightarrow g_{x}$ from $\operatorname{Hom}_{R}(M, M)$ to $\operatorname{Hom}_{R_{x}}\left(M_{x}, M_{x}\right)$ is the composite $\operatorname{Hom}_{R}(M, M) \rightarrow \operatorname{Hom}_{R}(M, M) \otimes_{R} R_{x}=\operatorname{Hom}_{R}(M, M)_{x} \rightarrow \operatorname{Hom}_{R_{x}}\left(M_{x}, M_{x}\right) ;$ this last map is a monomorphism when $M$ is finitely generated and is an isomorphism when $M$ is finitely generated and projective.

Proof. If $F$ is free on a finite number of generators and $F \rightarrow M$ is an epimorphism, then we have induced monomorphisms

$$
\begin{aligned}
& \operatorname{Hom}_{R}(M, M) \rightarrow \operatorname{Hom}_{R}(F, M) \\
& \operatorname{Hom}_{R}(M, M) \otimes_{R} R_{x} \rightarrow \operatorname{Hom}_{R}(F, M) \otimes R_{x}
\end{aligned}
$$

(since $R_{x}$ is flat) and isomorphisms

$$
\operatorname{Hom}_{R}(F, M) \otimes_{R} R_{x} \cong \operatorname{Hom}_{R}\left(F, M_{x}\right) \cong \operatorname{Hom}_{R_{x}}\left(F_{x}, M_{x}\right)
$$

the first resulting from the fact that $F$ is a finite product of $R$ 's. Similarly, $F_{x} \rightarrow M_{x}$ is an epimorphism and induces a monomorphism $\operatorname{Hom}_{R_{x}}\left(M_{x}, M_{x}\right)$ $\rightarrow \operatorname{Hom}_{R_{x}}\left(F_{x}, M_{x}\right)$. Thus in the commutative diagram

$$
\begin{aligned}
\operatorname{Hom}_{R}(M, M) \otimes_{R} R_{x} & \rightarrow \operatorname{Hom}_{R}(F, M) \otimes_{R} R_{x} \\
\downarrow & \downarrow \\
\operatorname{Hom}_{R_{x}}\left(M_{x}, M_{x}\right) & \rightarrow \operatorname{Hom}_{R_{x}}\left(F_{x}, M_{x}\right)
\end{aligned}
$$

three of the four arrows are monomorphisms. It follows that the fourth is, too. The isomorphism statement is standard [CE, VI. 4. 1. 3].

(2. 8) Every finite set of elements in $M x$ is contained in $M e$ for some $e \in x$. If $m_{x}=0$ for all $m$ in a finite subset of $M$, then $m(1-e)=0$ for some $e \in x$ and for all $m$ in this subset.

Proof. Such a finite set is contained in $\Sigma M e_{i}$ for some finite subset $\left\{e_{i}\right\} \subset x$. If $e$ is the union of the $e_{i}$, then $e \in x$ and $e_{i} \in R e$ so $\Sigma M e_{i} \subset M e$ (cf. $[\mathrm{P}, 1.6])$. The last part of $(2.8)$ merely asserts $M e(1-e)=0$.

(2.9) Let $a$ and $b$ be elements of $M$ and $a_{x}=b_{x}$ at one point of $X$. Then $a_{y}=b_{y}$ for all $y$ in some neighborhood $U_{e}$ of $x$, that is, $a(1-e)=b(1-e)$ for some $e \in x$. If $a_{x}=b_{x}$ for every $x$, then $a=b$.

Proof. If $a_{x}=b_{x}$ then (2.8) shows $(a-b)(1-e)=0$. If $a_{x}=b_{x}$ for every $x$ in $X$ then for each $x$ in $X$ we have $e \in x$ with $a(1-e)=b(1-e)$. Since $X$ is compact, it is covered by a finite number of the $U_{e}$, say 
$U_{e_{1}}, \cdots, U_{e_{n}}$. By (2.10) this means $1=\sum r_{i}\left(1-e_{i}\right)$ so that $a=\sum a r_{i}\left(1-e_{i}\right)$ $=\sum b r_{i}\left(1-e_{i}\right)=b$.

$$
\cup_{e \in A} U_{e}=X \text { implies } 1 \in \Sigma_{e \in A} R(1-e) \text {. }
$$

Proof. The hypothesis means every $x$ is in some $U_{e}$, that is every $x$ meets $A$, that is, the Boolean ideal generated by all $1-e$ with $e \in A$ is not contained in any $x$. This must then be the unit ideal, so that 1 is a linear combination in $B(R)$ of $\{1-e \mid e \in A\}$. Such a linear combination is also a linear combination in $R$ (the Boolean sum $e \oplus f$ is $e+f-e f$ ), proving (2. 10).

(2. 11) If $N$ is a submodule of $M$ and $N_{x}=M_{x}$ for all $x$ (note: since $R_{x}$ is flat over $R, N_{x} \subset M_{x}$ ), then $N=M$ (that is, $\oplus_{x \in X} R_{x}$ is faithfully flat).

If $M$ is finitely generated and $N_{x}=M_{x}$ for one $x$, then there is a neighborhood of $x$ such that $N_{y}=M_{y}$ for every $y$ in the neighborhood.

Proof. By reducing to $M / N$, we can assume $N=0$. If $a \in M$ and $a_{x}=0$ then $a \in M x$. If $M$ is finitely generated, (2.8) gives $e \in x$ such that $M=M e$. Then for every $y$ containing $e, M_{y}=0$. If $a_{x}=0$ for all $x$, then $a=0$ by (2.9).

We can lift idempotents:

(2. 12) Let $S$ be any R-algebra and $u$ any idempotent in $S_{x}$. Then there is an idempotent $v$ in $S$ such that $v_{x}=u$.

Proof. Lift $u$ to any element $w$ in $S$. Then $\left(w^{2}-w\right)_{x}=0$. By (2.9), $\left(w^{2}-w\right)(1-e)=0$ for some $e \in x$. This implies $v=w(1-e)$ is idempotent and $v_{x}=w_{x}-w_{x} e_{x}=w_{x}=u$.

(2. 13) $[\mathrm{P}, 4.4] R_{x}$ has no idempotents except 0 and 1.

Proof. If $u$ is idempotent in $R_{x}$ and $v$ is an extension to $R$ as in (2. 12) then either $v \in x$ or $1-v \in x$, by (2. 2a). Thus $u=0$ or 1 .

We can extend automorphisms:

(2.14) Suppose $S$ is an $R$-algebra that is finitely presented as an $R$-module, let $N$ be a finite subset of $S$ and let $g$ be an $R_{x}$-algebra automorphism of $S_{x}$ that is the identity on $N_{x}$. Then there is an R-algebra automorphism $h$ of $S$ such that $h$ is the identity on $N$ and $h_{x}=g$. 
Proof. Let $e_{1}, \cdots, e_{n}$ be $R$-module generators of $S$ whose relations are also finitely generated and let $\sum_{j} r_{p_{j}} e_{j}=0$ be a finite set of defining relations. Further, let $e_{i} e_{j}=\sum_{k} r_{i j k}^{\prime} e_{k}$ be the multiplication table of the $e$ 's, and let $n_{q}=\sum_{j} r^{\prime \prime}{ }_{q j} e_{j}$ be the elements of $N$. Then we are only required to find $f_{1}, \cdots, f_{n}$ in $S$, to serve as $h\left(e_{1}\right), \cdots, h\left(e_{n}\right)$, satisfying

$$
\begin{aligned}
& \sum_{j} r_{p j} f_{j}=0 \\
& f_{i} f_{j}-\sum_{k} r_{i j k}^{\prime} f_{k}=0 \\
& \sum_{j} r^{\prime \prime}{ }_{q j} f_{j}-\sum_{j} r_{q j}^{\prime \prime} e_{j}=0 .
\end{aligned}
$$

We know this finite set of equations is satisfied modulo $S x$ by $\left\{g\left(e_{i x}\right)\right\}$. Lift each $g\left(e_{i_{x}}\right)$ to any element $f_{i}^{\prime}$ in $S$ and consider the finite number of elements of $S$ that are the left hand sides of (2.15) with $f_{i}^{\prime}$ replacing $f_{i}$. Since these elements are 0 at $x$, they are all contained in Se for some $e$ in $x$ by (2.8). It follows that $\left(f_{i}^{\prime}\right)_{y}$ satisfies (2.15) for every $y$ in $X$ that contains $e$. Let $f_{i}=f_{i}^{\prime}(1-e)+e_{i} e$. Then for every $y$ in $X$, either $e \in y$ in which case $f_{i y}=\left(f_{i}{ }^{\prime}\right)_{y}$ satisfies (2.15), or $1-e \in y$ in which case $f_{i y}=e_{i y}$, which also satisfies (2.15). Thus with these $f_{i}$, the left hand sides of (2.15) are zero at every point of $X$, hence are zero (2.9).

(2.16) Theorem. Let $S$ be an R-algebra, finitely generated as an $R$-module and assume that the group of all $R_{x}$-algebra automorphisms of $S_{x}$ is finite, for each $x$ in $X$. Then every finite set of $R$-algebra automorphisms of $S$ generates a finite group (the automorphism group of $S$ is "locally finite").

Proof. Let $h_{1}, \cdots, h_{n}$ be the finite set of automorphisms of $S$, and $H$ the group they generate. Then for each $x, h_{1 x}, \cdots, h_{n x}$ satisfy enough relations to make $H_{x}$ a finite group. Let $m_{i}$ be these relations, that is, each $m_{i}$ is a monomial in the $h_{j}$ and the $h_{j}^{-1}$ and $m_{i}\left(h_{1 x}, \cdots, h_{n x}\right)=1$. This last means that the image of each $m_{i}\left(h_{1}, \cdots, h_{n}\right)$ under the $\operatorname{map} \operatorname{Hom}_{R}(S, S)$ $\rightarrow \operatorname{Hom}_{R_{x}}\left(S_{x}, S_{x}\right)$ is the identity. By (2.7) so is the image in $\operatorname{Hom}_{R}(S, S) \otimes R_{x}$. Therefore $m_{i}\left(h_{1}, \cdots, h_{n}\right)_{x}=1_{x}$. By (2.8) with $M=\operatorname{Hom}_{R}(S, S)$, we have $m_{i}\left(h_{1}, \cdots, h_{n}\right)(1-e)=1-e$ for some $e$ in $x$. Since the $h_{j}$ are all the identity on $e$, this means that the $h_{j}$ induce on $S / S e$ automorphisms that satisfy enough relations (namely the $m_{i}$ ) to generate a finite group. For every $x$ in $X$, we have produced a neighborhood $U_{e}$ of $x$, such that $H$ induces on $S / S e$ a finite group $H_{e}$. By the compactness of $X$, we cover $X$ 
with finitely many of these neighborhoods $\left\{U_{e} \mid e \in A\right\}$. Then $H$ is embedded in the finite product of the finite groups $H_{e}, e \in A$, because if $h$ in $H$ induces the identity on every $S / S e$ then $h_{x}$ is the identity on every $S_{x}=S / S x$ (since every $x$ contains some $e$ in $A$ ); from (2.7) $h \rightarrow 1$ under $\operatorname{Hom}_{R}(S, S)$ $\rightarrow \operatorname{Hom}_{R}(S, S)_{x} ;(2.9)$ then implies $h=1$. Thus $H$ is finite.

(2.17) If $S$ is an $R$-algebra and $F$ is a finite group of $R$-algebra automorphisms of $S$, then for every $x$ in $X,\left(S^{F}\right)_{x}=\left(S_{x}\right)^{F_{x}}$ where $S^{F}$ denotes the fixed ring under $F$ (3. 3).

Proof. The inclusion $\subset$ is trivial. If $u \in S_{x}$ and $f_{x}(u)=u$ for every $f$ in $F$, lift $u$ to an element $v$ in $S$ and have $(f(v)-v)_{x}=0$ for all (finitely many) $f$ in $F$. By (2.8), $f(v)(1-e)=v(1-e)$ for some $e$ in $x$. If $s=v(1-e)$, we have $s \in S^{F}$ and $s_{x}=v_{x}=u$, which completes the proof.

\section{Galois theory.}

Henceforth we consider a ring extension $R \subset S$ satisfying the following hypotheses.

(3. 1) Definition. A commutative $R$-algebra $S$ is said to be weakly Galois (over $R$ ) provided

(3. 1a) $S$ is a finitely generated, faithful, projective $R$-module, and a separable $R$-algebra.

(3. 1b) The $S$-module $\operatorname{Hom}_{R}(S, S)$ is generated by $R$-algebra automorphisms of $S$. If $\rho: S \rightarrow \operatorname{Hom}_{R}(S, S)$ is the usual regular representation of $S, \rho(s)(t)=s t$, then (3.1b) may be phrased thus:

(3.1c) $\rho(S) G=\operatorname{Hom}_{R}(S, S)$.

See also (3.6) and (3.15) for equivalent conditions.

(3. 2) If $S$ is weakly Galois over $R$, then for every $x$ in $X, S_{x}$ is weakly Galois over $R_{x}$.

Proof. (3. 1a, b) are inherited under localizations.

(3.3) Definition. If $S$ is a ring and $H$ is a set of automorphisms of $S$, we use $S^{H}$ to denote the fixed ring $\{s \in S \mid h(s)=s$ for every $h$ in $H\}$. If $T$ is a subring of $S, \operatorname{Aut}_{T}(S)$ will denote the group of all automorphisms of $S$ that are the identity on $T$. 
(3. 4) If $S$ is weakly Galois over $R$ and $G=\operatorname{Aut}_{R}(S)$, then $S^{G}=R$.

If $s \in S^{G}$, then $\rho(s)$ commutes with $G$ in $\operatorname{Hom}_{R}(S, S)$. Since $S$ is commutative, $\rho(s)$ commutes with $\rho(S)$. Hence, by (3.1c), $\rho(s)$ is in the center of $\operatorname{Hom}_{R}(S, S)$, which, because of (3.1a) is $\rho(R)$. Hence $s \in R$.

In Section 4 we give an example to show that the converse of (3.4) fails, even in the presence of (3.1a). However, (3.6), which gives an equivalent formulation of "weakly Galois", may be considered a kind of converse. The converse holds in the following form in the absence of idempotents:

(3. 5) Suppose $R$ has no idempotents but 0 and 1 . If $S$ satisfies (3.1a) and if $S^{H}=R$ for some subgroup $H$ of $G$, then $\rho(S) H=\operatorname{Hom}_{R}(S, S)$; in particular, the Galois theory in [VZ] applies if and only if $S$ is weakly Galois over $R$.

Proof. If $e_{1}, \cdots, e_{n}$ is the set of minimal idempotents in $S$, then $H$ must be transitive on this set and $S e_{j}$ must be $H_{j}$-Galois over $R$ [VZ, 1. 3] where $H_{j}$ is the subgroup of $H$ that sends $S e_{j}$ into itself. If $\rho_{j}$ is the regular representation of $S e_{j}$, then this implies $\operatorname{Hom}_{R}\left(S e_{j}, S e_{j}\right)=\rho_{j}\left(S e_{j}\right) H_{j}$. If $h_{i j}$ is an element of $H$ sending $e_{i}$ to $e_{j}$, then $\operatorname{Hom}_{R}(S, S)=\oplus_{i, j} \operatorname{Hom}_{R}\left(S e_{i}, S e_{j}\right)$ $=\oplus_{i, j} \operatorname{Hom}_{R}\left(S e_{j}, S e_{j}\right) h_{i j}=\oplus_{i, j} \rho_{j}\left(S e_{j}\right) H_{j} h_{i j} \subset \rho(S) H$.

(3. 6) Theorem. Let $S$ be an R-algebra satisfying (3.1a). Then $S$ is weakly Galois over $R$ if and only if there is a finite group (equivalently, a finite set) of automorphisms of $S$ having fixed ring $R$.

Proof. Assume $S$ is weakly Galois. By (3.2) and (3.5), the Galois theory in [VZ] applies to $S_{x}$ for each $x$ in $X$. That is, $\operatorname{Aut}_{R_{x}}\left(S_{x}\right)$ is a finite group with fixed ring $R_{x}$. Use $(2.14)$ to extend this finite group to automorphisms $h_{1}, \cdots, h_{n}$ of $S$. These will generate a finite group $H$, by (2. 16). By (3. 5), $(\rho(S) H)_{x}=\rho_{x}\left(S_{x}\right) H_{x}=\operatorname{Hom}_{R_{x}}\left(S_{x}, S_{x}\right)=\operatorname{Hom}_{R}(S, S)_{x} \quad$ (of course, $\rho_{x}$ is the regular representation of $S_{x}$ ). It follows by (2.11) that $\rho_{y}\left(S_{y}\right) H_{y}=(\rho(S) H)_{y}=\operatorname{Hom}_{R}(S, S)_{y}$ for all $y$ in a neighborhood of $x$. In particular, $\left(S_{y}\right)^{H_{y}}=R_{y}$ by (3. 4). For each $x$ we have a finite group $H$ and a neighborhood of $x$ such that at each point $y$ in the neighborhood, $\left(S_{y}\right)^{H_{y}}=R_{y}$. By compactness, we get a finite number of these neighborhoods covering $X$ and a finite number of $H^{\prime} s$ which then generate a finite group $F$ with $\left(S_{x}\right)^{F_{x}}=R_{x}$ at every point $x . \quad$ By (2.17) and (2.11), $S^{F}=R$. 
Conversely, if $F$ is finite and $S^{F}=R$, then for every $x, R_{x}=\left(S^{F}\right)_{x}=S_{x}{ }^{F_{x}}$, which by (3.5) and (2.7) implies $\rho_{x}\left(S_{x}\right) F_{x}=\operatorname{Hom}_{R_{x}}\left(S_{x}, S_{x}\right)=\operatorname{Hom}_{R}(S, S)_{x}$. It then follows from (2.11) that $\rho(S) F=\operatorname{Hom}_{R}(S, S)$, proving $S$ is weakly Galois.

(3. 7) Definition. If $H$ is a group of automorphisms of $S$, the closure of $H$ is the set of all automorphisms $g$ that satisfy either of the following equivalent conditions :

(3. 7a) For each $x$ in $X$ and each minimal idempotent $f$ in $S_{x}, \rho_{x}(f) g_{x}=$ $\rho_{x}(f) h_{x}$ for some $h$ in $H$.

(3. 7b) For some set $\left\{E_{i}\right\}$ of idempotents in $S$ with $\cup E_{i}=1, \rho\left(E_{i}\right) g=\rho\left(E_{i}\right) h_{i}$ for some $h_{i}$ in $H$ (all $i$ ).

$H$ is closed if it equals its own closure.

The condition $\cup E_{i}=1$ in $(3.7 \mathrm{~b})$, of course, is a statement in the Boolean algebra $B(S)$; it means that if $E$ is an idempotent in $S$ and $E E_{i}=E_{i}$ for all $i$, then $E=1$. However, for comparison between (3.7a) and (3.7b), another condition is more convenient: for every $x$ in $X$ and for every minimal idempotent $f$ in $S_{x}$ there is some $i$ such that $E_{i x} \geq f$, that is $E_{i x} f=f$. The proof that these two conditions are equivalent is not difficult and runs along our typical line of extension arguments.

We now prove the equivalence of (3.7a) and (3.7b). If $g$ satisfies (3. 7a), lift each $f$ in each $S_{x}$ to an idempotent $E_{f}$ in $S$ by (2.12), and consider the two elements $u=\rho\left(E_{f}\right) g$ and $v=\rho\left(E_{f}\right) h$ in $\operatorname{Hom}_{R}(S, S)$, where $h$ is the element of $H$ given in (3.7a) such that $\rho_{x}(f) g_{x}=\rho_{x}(f) h_{x}$. By (2. 7) we conclude the images of $u$ and $v$ in $\operatorname{Hom}_{R}(S, S)_{x}$ are equal.

By (2. 9), $u(1-e)=v(1-e)$ for some $e \in x$. Write $E_{f}^{\prime}=(1-e) E_{f}$. Then $\rho\left(E_{f}^{\prime}\right) g=\rho\left(E_{f}^{\prime}\right) h$ and $\left(E_{f}^{\prime}\right)_{x}=(1-e)_{x}\left(E_{f}\right)_{x}=f$.

Conversely, for each minimal idempotent $f$ in $S_{x}$, choose an $E_{i}$ such that $E_{i x}=f$. Then $\rho\left(E_{i}\right) g=\rho\left(E_{i}\right) h_{i}$ implies $\rho_{x}\left(E_{i x}\right) g_{x}=\rho_{x}\left(E_{i x}\right) h_{i x}$, as desired.

(3. 8) Theorem. Let $S$ be a weakly Galois R-algebra. Then the usual Galois correspondence (3.3) is one-to-one between the set of all separable subalgebras of $S$ and the set of all subgroups $H$ of the automorphism group of $S$ that satisfy (3. 8c) below, or, equivalently, that satisfy (3.8a) and (3.8b):

2) Added August 12, 1968: A. Magid has improved (3.7b) to $\rho\left(E_{i}\right) g=\rho\left(E_{i}\right) h_{i}$ for some finite set of orthogonal idempotents $E_{i}$ with $\sum E_{i}=1$ and some $h_{i}$ in $H$. 
(3. 8a) For some finite subgroup $F$ of $H, S^{F}=S^{H}$.

(3. 8b) $H$ is closed in the sense of (3.7).

(3. 8c) $H$ is the closure of some finite set (equivalently, some finite group) of automorphisms of $S$.

Proof. We need to show five things:

(3. 9a) If $T$ is separable and $H=\operatorname{Aut}_{T}(S)$, then $H$ satisfies (3.8a) and (3. $8 \mathrm{~b})$.

(3. 9b) If $H$ is a subgroup of the automorphism group of $S$ and $H$ satisfies (3.8a) and (3.8b), then $S^{H}$ is separable over $R$.

(3. 9c) If $T$ is separable and $H=\operatorname{Aut}_{T}(S)$, then $T=S^{H}$.

(3. 9d) If $H$ satisfies (3.8a) and (3.8b) and $T=S^{H}$, then $\operatorname{Aut}_{T}(S)=H$.

(3. 9e) (3. 8c) is equivalent to (3.8a) and (3.8b).

We begin with a stronger version of (3.9b).

(3.10) If $F$ is a finite subgroup of $G$, then $S^{F}$ is a separable subalgebra of $S$.

Proof. First, we argue that if $T=S^{F}$, then $T$ is flat over $R$ : For every $x$ in $X, T_{x}=S_{x}^{F_{x}}$ by (2.17), but this is separable over $R_{x}$ by [VZ, Theorem p. 731]. Hence $T_{x}$ is also projective and therefore flat over $R_{x}$. For every $R$-module $A$ and every $x,\left(\operatorname{Tor}^{R}(T, A)\right)_{x}=\operatorname{Tor}_{x}^{R_{x}}\left(T_{x}, A_{x}\right)=0 . \quad$ This implies $\operatorname{Tor}^{R}(T, A)=0$ by (2. 11).

Since $T$ and $S$ are both flat over $R$, the mapping $T \otimes T \rightarrow S \otimes S$ is a monomorphism, and we may identify $T \otimes T$ with its image in $S \otimes S$. To prove $T$ is separable, then, it suffices to produce an element $f$ in $S \otimes S$ with the following properties

$$
f \in T \otimes T
$$

$$
\begin{gathered}
(1 \otimes t-t \otimes 1) f=0 \text { for all } t \text { in } T \\
\mu(f)=1
\end{gathered}
$$

where $\mu: S \otimes S \rightarrow S$ is the multiplication map, for then $T \otimes T \rightarrow T$ will split as $(T \otimes T)$-module map, since a reverse map may be defined by the condition that it send 1 to $f$. This splitting implies $T$ is separable.

Since $S$ is separable $\mu$ splits, and the image of 1 under this reverse map is an element $e$ in $S \otimes S$ having the properties $(1 \otimes s-s \otimes 1) e=0$ for 
all $s$ in $S$ and $\mu(e)=1$. This $e$ is unique due to the commutativity of $S$; since for every $g$ in $G,(g \otimes g)(e)$ has these properties, we have $(g \otimes g)(e)=e$.

Now, for each $h$ in $F$, write $e_{h}=(h \otimes 1)(e)$. Since $h \otimes 1$ is an $(R \otimes S)$ algebra automorphism of $S \otimes S,(1 \otimes s-h(s) \otimes 1) e_{h}=0$ for all $s$ in $S$. In particular, $(1 \otimes t-t \otimes 1) e_{h}=0$ for all $t$ in $T$.

Let $f$ denote the union of the $e_{h}$,

$$
f=1-\Pi_{h \in F}\left(1-e_{h}\right) \text {. }
$$

Then (3.11b) holds since it holds for each $e_{h}$, and (3.11c) holds because $\mu(1-e)=0$ and so $\mu\left(\Pi\left(1-e_{h}\right)\right)=0$. It remains to show that $f \in T \otimes T$. It is clear that $f \in(S \otimes S)^{F \otimes F}$ because for all $h, h^{\prime}, h^{\prime \prime}$ in $F$,

$$
\begin{aligned}
\left(h^{\prime} \otimes h^{\prime \prime}\right)\left(e_{h}\right) & =\left(h^{\prime} h \otimes h^{\prime \prime}\right)(e)= \\
& =\left(h^{\prime} h h^{\prime \prime-1} \otimes 1\right)\left(h^{\prime \prime} \otimes h^{\prime \prime}\right)(e)=\left(h^{\prime} h h^{\prime \prime-1} \otimes 1\right)(e)=e_{h^{\prime} h h^{\prime \prime}-1}
\end{aligned}
$$

so $h^{\prime} \otimes h^{\prime \prime}$ permutes the $e_{h}$ 's, and will leave $f$ fixed. Therefore, it remains to show $(S \otimes S)^{F \otimes F}=T \otimes T$. If $h \in F$ then $S^{\{h\}}=\operatorname{Ker}(1-h: S \rightarrow S)$, so, since $S$ is flat over $R, S \otimes S^{\{h\}}=\operatorname{Ker}(1-1 \otimes h)=(S \otimes S)^{\{1 \otimes h\}}$. Further, if $S_{1}, \cdots, S_{n}$ are $R$-submodules of $S$, then $S \otimes\left(\cap_{i=1}^{n} S_{i}\right)=\cap\left(S \otimes S_{i}\right)$ (since $\cap S_{i}=\operatorname{Ker}\left(S \rightarrow \Pi\left(S / S_{i}\right)\right)$ and tensoring with a flat module $S$ preserves kernels and finite products of $R$-modules). Hence $S \otimes S^{F}=(S \otimes S)^{1 \otimes F}$. In the same way, since $T$ is flat over $R,(S \otimes T)^{F \otimes 1}=S^{F} \otimes T$. Combining, $(S \otimes S)^{F \otimes F}=\left((S \otimes S)^{1 \otimes F}\right)^{F \otimes 1}=(S \otimes T)^{F \otimes 1}=T \otimes T$.

In view of (3.4), the following is enough to prove (3.9c):

(3. 12) If $S$ is weakly Galois over $R$ and if $T$ is a separable subalgebra of $S$ then $S$ is weakly Galois over $T$.

Proof. If $T$ is separable, then $T$ is finitely generated as an $R$-module $[\mathrm{AB}, 4.7$ and 4.8$]$, say by a finite set $N$. Use (2.14) to conclude that $H=\operatorname{Aut}_{T}(S)$ induces on $S_{x}$ the full automorphism group of $S_{x}$ over $T_{x}$, for each $x$ in $X$. Then by (3.5) and (2.7) $\rho_{x}\left(S_{x}\right) H_{x}=\operatorname{Hom}_{T_{x}}\left(S_{x}, S_{x}\right)=\operatorname{Hom}_{T}(S, S)_{x}$ (this last equality needs the fact that $S$ is finitely generated and projective over $T$, which also comes from $[\mathrm{AB}, 4.8])$. It then follows from (2. 11) that $\rho(S) H=\operatorname{Hom}_{T}(S, S)$.

We have proved part of (3.9a), namely (3.8a), because (3.12) asserts that $S$ is weakly Galois over $T$ and (3.6) then implies that $S^{F}=T$ for some finite subgroup $F$ of $H$. Combined with (3.9c), this gives (3.8a). 
To complete the proof of (3.9a), it suffices to show

(3. 13) For every subalgebra $T$ of $S, \operatorname{Aut}_{T}(S)$ is closed.

Proof. If $g$ is an automorphism such that, for every $x$ in $X$ and every minimal idempotent $f$ in $S_{x}, \rho_{x}(f) g_{x}=\rho_{x}(f) h_{x}$ for some $h$ in $\operatorname{Aut}_{T}(S)$, then for every $t$ in $T, f g_{x}\left(t_{x}\right)=\rho_{x}(f) g_{x}\left(t_{x}\right)=\rho_{x}(f) h_{x}\left(t_{x}\right)=f t_{x}$. Since the sum of all $f$ in $S_{x}$ is 1 , we have $g_{x}\left(t_{x}\right)=t_{x}$ or $g(t)_{x}=t_{x}$ for every $x$. By (2. 9), $g(t)=t$ and so $g \in \operatorname{Aut}_{T}(S)$.

(3. 14) If $F$ is a finite group of automorphisms of $S$ and if $T=S^{F}$, then $\operatorname{Aut}_{T}(S)$ is contained in the closure of $F$.

Proof. Suppose $g \in \operatorname{Aut}_{T}(S)$. Then for every $x$ in $X g_{x}$ is an automorphism of $S_{x}$ that is the identity on $T_{x}$, and $T_{x}=S_{x}^{F_{x}}$, according to (2. 17). By [VZ, Theorem] $g_{x}$ is in the "fat group generated by $F_{x}$ ", that is, for every minimal idempotent $f$ in $S_{x}, \rho_{x}(f) g_{x}=\rho_{x}(f) h_{x}$ for some $h_{x}$ in $F_{x}$. This is the same as saying $g$ is in the closure of $F$.

This completes the proof of (3.14). (3.9d) follows immediately: If $H$ is any group satisfying (3.8a) and (3.8b) and if $T=S^{H}=S^{F}$ with $F$ a finite subgroup of $H$, then since the closure operation preserves inclusions, the closure of $F$ is contained in $H$. But $T=S^{H}$ implies that $H \subset \operatorname{Aut}_{T}(S)$, and we have just proved in (3.14) that $\operatorname{Aut}_{T}(S)$ is contained in the closure of $F$. Hence $H=\operatorname{Aut}_{T}(S)=$ closure of $F$. Notice that we have simultaneously proved (3.9d) and half of (3.9e): (3.8a) and (3.8b) imply (3.8c).

To complete the proof of (3.9e), suppose $H$ satisfies (3. 8c), write $H=$ the closure of $F$ with $F$ finite, and set $T=S^{F}$. According to (3.14), $\operatorname{Aut}_{T}(S) \subset H$. By (3.13) and because $F \subset \operatorname{Aut}_{T}(S), \quad H \subset \operatorname{Aut}_{T}(S)$, which means $H=\operatorname{Aut}_{T}(S)$. Since $T$ is separable by (3.10), we may use (3.9c) to conclude that $T=S^{H}$, so that $H$ satisfies (3.8a). It is a triviality that every closure is closed, so $H$ satisfies (3. 8b).

We can now compare our weakly Galois hypothesis with that of Kreimer. His condition is this:

(3. 15) There exists a finite set of orthogonal idempotents $\left\{e_{1}, \cdots, e_{n}\right\}$ in $R$ with $\sum e_{i}=1$ and with $S e_{i}$ Galois over $R e_{i}$ for each $i$.

This clearly implies weakly Galois, but we shall also prove the converse. First, if $S$ is weakly Galois over $R$ we show that $S_{n}$ is Galois over $R_{n}$ 
for each $x$ in $X$. If $S_{x}=\Pi S_{i}$ is a decomposition of $S_{x}$ into indecomposable $R_{x}$-algebras, then all the $S_{i}$ must be isomorphic, else the sum of the identity elements of those $S_{i}$ that are in one isomorphism class will be a proper idempotent in $S_{x}$ that is invariant under all automorphisms, hence will be a proper idempotent in $R_{x}$, contradicting (2.13). Now choose one isomorphism $\alpha_{i}: S_{1} \rightarrow S_{i}$ for each $i$ and construct an automorphism $\alpha$ of $S_{x}$ by demanding that $\alpha$ restricted to $S_{i}$ be $\alpha_{i+1} \circ \alpha_{i}^{-1}$ for $i<n$ and $\alpha$ restricted to $S_{n}$ be $\alpha_{n}^{-1}$. Let $H$ be the cyclic group generated by $\alpha$ and let $G_{x}$ be the product of $H$ and the automorphism group of $S_{1}$ over $R_{x}$. Then $S_{x}$ will be $G_{x}$-Galois over $R_{x}$ (the elements of $G_{x}$ are strongly distinct in the sense of $[\mathrm{CHR}, 1.1])$.

Now lift $G_{x}$ to a set of automorphisms of $S$ by (2.14). The multiplication table that holds at $x$ will hold in a neighborhood $U_{e}$ of $x$, so this lifted set will be a group in $U_{e}$, isomorphic to $G_{x}$. If $S e[G]$ denotes the usual crossed product (with trivial factor set) of $G$ over the ring $\mathrm{Se}$, we have the homomorphism $\theta: S e[G] \rightarrow \operatorname{Hom}_{R e}(S e, S e)$. We know this induces an isomorphism at $x$, since the definition of " $S_{x}$ is $G_{x}$-Galois over $R_{x}$ " requires $S_{x}\left[G_{x}\right] \rightarrow \operatorname{Hom}_{R_{x}}\left(S_{x}, S_{x}\right)$ be an isomorphism and the localization at $x$ of $\mathrm{Se}[G]$ is $S_{x}\left[G_{x}\right]$ and of $\operatorname{Hom}_{R e}(S e, S e)$ is $\operatorname{Hom}_{R_{x}}\left(S_{x}, S_{x}\right)$. If $C$ denotes the cokernel of $\theta$, then $C_{x}=0$; but $C$ is finitely generated over $R e$ since $\operatorname{Hom}_{R e}(S e, S e)$ is, so $C$ is zero in some neighborhood of $x$, that is $C e^{\prime}=0$ for some $e^{\prime}$ with $x \in U_{e^{\prime}} \subset U_{e}$. Hence $\theta^{\prime}: S e^{\prime}[G] \rightarrow \operatorname{Hom}_{R e^{\prime}}\left(S e^{\prime}, S e^{\prime}\right)$ is an epimorphism. But $\operatorname{Hom}_{R e^{\prime}}\left(S e^{\prime}, S e^{\prime}\right)$ is projective over $R e^{\prime}$, so the kernel of $\theta^{\prime}$ is a direct summand in $S e^{\prime}[G]$, hence finitely generated over $R e^{\prime}$. The same localization argument then shows that this kernel is also zero in some smaller neighborhood of $x$. Thus for every $x$ in $X$ there is a neighborhood $U_{e^{\prime \prime}}$ of $x$ such that $S e^{\prime \prime}[G] \rightarrow \operatorname{Hom}_{R e^{\prime \prime}}\left(S e^{\prime \prime}, S e^{\prime \prime}\right)$ is an isomorphism, that is $S e^{\prime \prime}$ is $G$-Galois over $R e^{\prime \prime}$. A finite number of neighborhoods cover $X$. Using the usual Boolean operations and noticing that if $S e$ is $G$-Galois over $R e$, then $S e^{\prime}$ is $G$-Galois over $R e^{\prime}$ for every $e^{\prime}$ with $U_{e^{\prime}} \subset U_{e}$, we can arrange that these neighborhoods $U_{e_{1}}, \cdots, U_{e_{n}}$ are disjoint. Then $\left\{e_{1}, \cdots, e_{n}\right\}$ is the required set of idempotents.

\section{Two examples.}

First we show that in the Galois correspondence, we must restrict ourselves to groups satisfying $(3.8 \mathrm{a})$ and $(3.8 \mathrm{~b})$; the latter closure condition is not enough by itself. 
Let $X$ be $\{1,1 / 2,1 / 3, \cdots, 1 / n, \cdots, 0\}$ with its relative topology as a subset of the real line and let $W$ be the ring of continuous functions from $X$ to the discrete field $Q$ of rational numbers. In other words, $W$ is the result of adjoining an identity to a countable ring-direct sum of copies of $Q$. The Boolean spectrum of $W$ is indeed $X$ and $w_{x}=w(x)$ for each $w$ in $W$. Let $K$ be a Galois extension field of $Q$ with Galois group $G$ and let $V$ be the ring of continuous functions from $X$ to $K$. Then $V$ is weakly Galois over $W$ since $V=K \otimes_{Q} W$ and $V^{(G \otimes 1)}=W$ (in fact, the extension is even strongly Galois). Consider the subgroup $H$ of $\operatorname{Aut}_{W}(V)$ consisting of all automorphisms $g$ such that $g$ is the identity at the point 0 in $X$. Then $H$ is closed, $H \neq \operatorname{Aut}_{W}(V)$, but $V^{H}=W$ because if $v \notin W$ then $v_{x} \in Q$ for some $x$ and hence for some $x \neq 0$; and $H$ contains an automorphism $h$ such that $h_{y}=$ identity for all $y \neq x$ and $h_{x}\left(v_{x}\right) \neq v_{x}$ so that $h(v) \neq v$. This shows that $H$ and $\operatorname{Aut}_{W}(V)$ cannot both fit into a Galois correspondence.

The second example shows that without assuming $S$ is weakly Galois, the partial assumptions (3.1a) and $S^{a}=R$ for some $G$ are not sufficient to carry out our Galois theory.

Let $W$ be as above, let $e_{n}$ denote the idempotent characteristic function of the one-point set $\{1 / n\}$ and let $R$ be $W[t, u] / I$ with $t$ and $u$ indeterminates over $W$ and $I$ the ideal generated by $t u-1$ and all $t e_{n}+n^{4} e_{n}$ for $n=1,2, \cdots$ Let $\alpha$ denote the residue class of $t$ in $R$. The Boolean spectrum of $R$ is still $X, R_{x}=Q$ for all $x \neq 0$ and $R_{0}=Q\left[t, t^{-1}\right]$ with $t=\alpha_{x}$ indeterminate over $Q$. Let $S$ be the result of adjoining a fourth root $\beta$ of $\alpha$ to $R$, so $S=R[v] /\left(v^{4}-\alpha\right)$. Then $S$ has a free basis $1, \beta, \beta^{2}$, $\beta^{3}$ over $R$ and is separable because $e=(1 / 4)\left(1 \otimes 1+\beta \otimes \beta^{-1}+\beta^{2} \otimes \beta^{-2}+\beta^{3} \otimes \beta^{-3}\right)$ is an element of $S \otimes_{R} S$ satisfying $\mu(e)=1$ and $e(\beta \otimes 1-1 \otimes \beta)=0$ so $e(x \otimes 1-1 \otimes x)=0$ for all $x$ in $S$. We now show $S^{G}=R$. If $x \neq 0$ then $S_{x}=Q\left[\sqrt[4]{-n^{4}}\right]=Q[\sqrt[4]{-1}]$, a Galois extension field of $R_{x}=Q$. Thus any element $s$ of $S$ that is invariant under all automorphisms of $S$ will have $s_{x} \in R_{x}$ for all $x \neq 0$. To show that in fact $s \in R$, write $s=a+b \beta+c \beta^{2}+d \beta^{3}$ with $a, b, c, d \in R$. Since $s_{x}=a_{x}+b_{x} \beta_{x}+c_{x} \beta_{x}^{2}+d_{x} \beta_{x}^{3} \in R_{x}$, we must have $b_{x}=c_{x}=d_{x}=0$ for all $x \neq 0$. By (2.9), if we show $b_{0}=c_{0}=d_{0}=0$ then $b=c=d=0$ and $s \in R$. Now $b=\sum_{i=m}^{n} a_{i} \alpha^{i}$ with $a_{i} \in W$ and $b_{x}=\sum a_{i}(x) \alpha_{x}{ }^{i}=0$ for all $x \neq 0$. Since $a_{0}, \cdots, a_{n}$ are continuous functions, all $a_{i}(x)$ are constant $\left(=a_{i}(0)\right)$ on some neighborhood of 0 . Thus we have a single polynomial $\sum a_{i}(0) t^{i}$ in $Q\left[t, t^{-1}\right]$ with infinitely many roots, namely $\alpha_{x}=-n^{4}$ for all integers $n$. 
This polynomial must be 0 , so $a_{i}(0)=0$ for all $i$. Thus $b_{0}=0$. Similarly, $c_{0}=d_{0}=0$ and we have proved $S^{G}=R$.

On the other hand, we show that $S_{0} a_{0} \neq R_{0}$ which, by (2. 17) and (3.6), show that $S$ is not weakly Galois over $R$ and also shows that our technique of reducing to the case of no idempotents will not work here. As we said, $R_{0}=Q\left[t, t^{-1}\right]$ with $t$ indeterminate over $Q$; and $S_{0}=R_{0}\left[\beta_{0}\right]$ with $\beta_{0}^{4}=t$.

Then $S_{0}$ is contained in the rational function field $Q\left(\beta_{0}\right) \quad\left(\beta_{0}\right.$ is indeterminate over $Q$ ) and contains no fourth roots of 1 except \pm 1 . If $g$ is an $R_{0}$-algebra automorphism of $S_{0}$, it carries $\beta_{0}$ to another fourth root of $t$, which must be $\beta_{0}$ times a fourth root of 1 . Hence $g\left(\beta_{0}\right)= \pm \beta_{0}$ and $g\left(\beta_{0}{ }^{2}\right)=\beta_{0}{ }^{2}$. Thus $\beta_{0}^{2}$ is in $S_{0}{ }^{G_{0}}$ but not in $R_{0}$.

\section{REFERENCES}

[AB] M. Auslander and D.A. Buchsbaum, On ramification theory in Noetherian rings, Amer. J. Math. 81 (1959) 749-765.

[AG] M. Auslander and O. Goldman, The Brauer group of a commutative ring, Trans. Amer. Math. Soc. 97 (1960) 367-409.

[CE] H. Cartan and S. Eilenberg, Homological Algebra, Princeton University Press, Princeton, 1956.

[CHR] S.U. Chase, D.K. Harrison, A. Rosenterg, Galois theory and Galois cohomology of commutative rings, Memoirs Amer. Math. Soc. No. 52, 1965.

[K] H.F. Kreimer, A note on the outer Galois theory of rings, to appear in Pacific J. Math.

[P] R.S. Pierce, Modules over commutative regular rings, Memoirs Amer. Math. Soc. No. 70, 1967.

[VZ] O.E. Villamayor and D. Zelinsky, Galois theory for rings with finitely many idempotents, Nagoya Math. J. 27 (1966) 721-731.

Northwestern University 